\title{
Quench dynamics and Hall response of interacting Chern insulators
}

\author{
Michael Schüler, ${ }^{1,2}$ Jan Carl Budich, ${ }^{3}$ and Philipp Werner ${ }^{1}$ \\ ${ }^{1}$ Department of Physics, University of Fribourg, 1700 Fribourg, Switzerland \\ ${ }^{2}$ Stanford Institute for Materials and Energy Sciences (SIMES), SLAC National Accelerator Laboratory, \\ Menlo Park, California 94025, USA \\ ${ }^{3}$ Institute of Theoretical Physics, Technische Universität Dresden, 01062 Dresden, Germany
}

(Received 30 November 2018; revised manuscript received 6 June 2019; published 2 July 2019)

\begin{abstract}
We study the coherent nonequilibrium dynamics of interacting two-dimensional systems after a quench from a trivial to a topological Chern insulator phase. While the many-body wave function is constrained to remain topologically trivial under local unitary evolution, we find that the Hall response of the system can dynamically approach a thermal value of the postquench Hamiltonian, even though the efficiency of this thermalization process is shown to strongly depend on the microscopic form of the interactions. Quite remarkably, the effective temperature of the steady-state Hall response can be arbitrarily tuned with the quench parameters. Our findings suggest a way of inducing and observing low-temperature topological phenomena in interacting ultracold atomic gases, where the considered quench scenario can be realized in current experimental setups.
\end{abstract}

DOI: 10.1103/PhysRevB.100.041101

Introduction. Recent experimental progress in realizing topological insulators in a nonequilibrium fashion in ultracold atomic gases [1-6] and periodically driven solids [7-9] raises fundamental questions regarding the quench dynamics of topological phases. Rather than preparing the ground state of the system of interest, the natural protocol in synthetic material systems is to start from a trivial initial state, and quench the (effective) Hamiltonian into a topological phase before observing the coherent dynamics of the system. In this scenario topological invariants of the many-body state, such as the (many-body) Chern number [10,11], are conserved (remain trivial) [12], as the postquench time evolution represents a local unitary transformation [13]. Nevertheless, several observables provide signatures of a change in the topological properties, including circular dichroism in photoabsorption [14-18], characteristic edge currents [19], and the nonequilibrium Hall effect [20-26]. In quenched noninteracting systems, the Hall response-as the archetype of a topological response property-typically exhibits long-lasting oscillations which may be reduced in certain cases by specifically designed quench protocols [27]. In open systems, where the aforementioned constraints on the temporal invariance of topological properties are absent, the Hall response may equilibrate due to extrinsic dephasing [23] or dissipation [15,28].

The purpose of this work is to consider the quench dynamics of closed interacting topological two-dimensional (2D) systems, where the time evolution is still unitary at a global level, while two-body scattering provides a source of intrinsic dissipation which may enable thermalization processes. In this coherent scenario, it is interesting to investigate which signatures of topology can dynamically equilibrate despite the manifestly trivial character of the time-evolved manybody state. Furthermore, thermalization in a closed quantum system is a complex process, and, depending on the allowed scattering processes, the system may be trapped in a longlived prethermal state [29-33]. For a one-dimensional system it has recently been shown [34] that the single-particle density matrix (SPDM) can thermalize toward an equilibrium state of a topologically nontrivial postquench Hamiltonian. However, the dynamical equilibration of natural observables for topological insulators, in particular two-particle quantities such as the Hall conductivity in 2D systems, has remained a largely unexplored question.

Below, we investigate the postquench dynamics of the Hall response in interacting 2D fermionic systems. With fully microscopic numerical simulations based on the time-dependent nonequilibrium Green's functions (NEGF) approach [35,36], we demonstrate that a dynamical equilibration to a thermal value of the Hall response is possible, if sufficiently many scattering channels are available, while thermalization bottlenecks related to the topological character of the considered quenches result in slow dynamics (see Fig. 1). Specifically, when considering a minimal model system for a Chern insulator, nearest-neighbor interactions are necessary to efficiently thermalize the Hall response, even though on-site interactions already render the system nonintegrable.

Topological Hubbard model. We study interacting topological insulator (TI) or Chern insulator models on a 2D square lattice with unit lattice constant, defined by the generic Hamiltonian

$$
\hat{H}=\hat{H}_{\mathrm{TI}}+\hat{H}_{\text {int }} \text {. }
$$

We consider two scenarios for the free Hamiltonian $\hat{H}_{\mathrm{TI}}$ :

(i) A spinless Chern insulator defined by $\hat{H}_{\mathrm{TI}}=$ $\sum_{\mathbf{k}} \hat{\mathbf{c}}_{\mathbf{k}}^{\dagger} \mathbf{h}(\mathbf{k}) \hat{\mathbf{c}}_{\mathbf{k}}$ with

$$
\begin{aligned}
\mathbf{h}(\mathbf{k})= & {\left[M-\cos \left(k_{x}\right)-\cos \left(k_{y}\right)\right] \sigma_{z}+\lambda \sin \left(k_{x}\right) \sigma_{x} } \\
& +\lambda \sin \left(k_{y}\right) \sigma_{y} .
\end{aligned}
$$

Here, the $\hat{\boldsymbol{c}}_{\mathbf{k}}^{(\dagger)}=\left(\hat{c}_{\mathbf{k} E}^{(\dagger)}, \hat{c}_{\mathbf{k} H}^{(\dagger)}\right)$ denote the fermionic annihilation (creation) operators with respect to the underlying two bipartite orbital bands, labeled by $E$ (electronlike) and $H$ (holelike), 

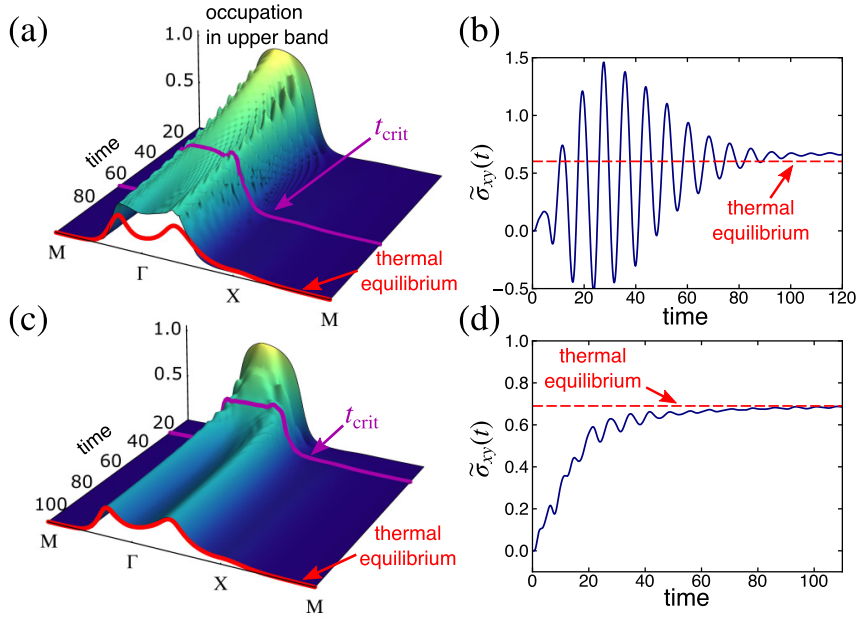

FIG. 1. Thermalization of occupation and Hall response. Upper panels: dynamics of the occupation in the upper band of the postquench Hamiltonian (a) and corresponding dynamical Hall response $\widetilde{\sigma}_{x y}(t)(\mathrm{b})$ in the interacting Chern insulator with local interactions only $(V=1)$. Lower panels: occupation dynamics (c) and buildup of the Hall response (d) in the Chern insulator including nonlocal interactions $\left(V_{0}=1, V_{1}=0.25 V_{0}\right)$. The results have been obtained within the GKBA, using a $N_{k}=220 \times 220$ grid sampling of the BZ.

respectively, in analogy to the original Bernevig-HughesZhang [37] model for HgTe, the $\sigma_{i}$ are the Pauli matrices in the orbital pseudospin space, and the lattice momentum $\mathbf{k}$ is defined in the first Brillouin zone (BZ).

(ii) A spinful quantum spin Hall insulator with timereversal symmetry defined by [37] $\hat{H}_{\mathrm{TI}}=\sum_{\mathbf{k}, \sigma} \hat{\mathbf{c}}_{\mathbf{k}, \sigma}^{\dagger} \mathbf{h}_{\sigma}(\mathbf{k}) \hat{\mathbf{c}}_{\mathbf{k} \sigma}$ with $\mathbf{h}_{\uparrow}(\mathbf{k})=\mathbf{h}(\mathbf{k}), \mathbf{h}_{\downarrow}(\mathbf{k})=[\mathbf{h}(-\mathbf{k})]^{*}$. In both cases, the system is a trivial band insulator for $M<-2$, while $-2<$ $M<0$ corresponds to a (spin) Chern insulator with (spin) Chern number $C=1$. In what follows, all energies (times) are measured in units of the hopping (inverse hopping).

Postquench dynamics with interactions. To gain insights into the dynamical manifestation of topological properties, the system is prepared in a low-temperature equilibrium state in the topologically trivial phase. At times $t=0_{+}$, the mass parameter $M$ is suddenly switched (continuous ramps of $M$ will be considered further below) to the topological regime and kept constant for $t>0$. To disentangle scattering processes in the postquench dynamics from the initial state, $\hat{H}_{\text {int }}$ is also switched on suddenly at $t=0_{+}$. This protocol can be realized experimentally in ultracold atomic gases by tuning a magnetic field in the vicinity of a Fano-Feshbach resonance $[38,39]$. We will consider weak to intermediate interparticle interactions in this work. There, in the absence of interaction-driven topological phase transitions [40-43], the topological character of the system is still determined by $\hat{H}_{\mathrm{TI}}$. This has been confirmed by inspecting the spectral function of the interaction system and by checking the quantized Hall response in equilibrium.

The time-dependent NEGF approach $[35,36,44]$ will be used to describe the correlated dynamics. This method is based on solving the Kadanoff-Baym equations (KBEs) for the single-particle Green's function, from which the SPDM $\boldsymbol{\rho}_{\sigma}(\mathbf{k} ; t)$ and thus all single-particle observables can be computed. We employ the second-Born approximation to the self-energy kernel, which has been shown to provide an excellent description of the electronic structure and dynamics for relatively weak interactions $[45,46]$.

Extrapolating to the thermodynamic limit requires a sufficiently large number of points in the discretized BZ. Due to the substantial numerical effort of solving the full KBEs, this poses a computational challenge. Invoking the generalized Kadanoff-Baym ansatz (GKBA) [47] is an additional approximation which reduces the numerical effort significantly. The GKBA has been shown to yield excellent results in the weakcoupling regime for single-particle observables [45,48-50]. How well nonequilibrium response properties are captured is less understood and will be addressed in the context of the Hall response below. Both the full KBE and the GKBA treatment conserve the total energy, and thus correctly capture an essential property of isolated systems. Therefore, comparing the energy of the system after the quench to the thermal equilibrium energies of the postquench interacting system allows one to determine the effective temperature $T_{\text {eff }}$ and corresponding thermalized observables.

Chern insulator with local interactions. As the first paradigmatic example we consider the case where $\hat{H}_{\mathrm{TI}}$ defines a spinless Chern insulator for $M<-2$. Restricting to local interactions, we consider the interaction term

$$
\hat{H}_{\mathrm{int}}=\frac{V}{2} \sum_{i} \sum_{\alpha \neq \alpha^{\prime}} \hat{n}_{i, \alpha} \hat{n}_{i, \alpha^{\prime}},
$$

where $i$ runs over all lattice sites, while $\alpha, \alpha^{\prime} \in\{E, H\}$. In the following, we fix $\lambda=0.4$ and consider the quench of the gap parameter $M_{\text {pre }} \rightarrow M_{\text {post }}$ with $M_{\text {pre }}=-3.5$ and $M_{\text {post }}=-1$.

Without band hybridization $(\lambda=0)$, the $E$ and $H$ bands possess a $U(1)$ symmetry, which results in individually conserved particle numbers $n_{E}$ and $n_{H}$. As a result, interorbital thermalization will be completely suppressed. In the case $\lambda>$ 0 and $C=1$, interorbital scattering in the lower and upper bands becomes possible, albeit only active close to the avoided crossings. Furthermore, the nonvanishing Chern number implies that even within the same Bloch band, there are states with opposite orbital character which are not connected by the intraorbital interaction. Therefore, thermalization can only proceed via higher-order scattering processes.

The expectation of slow thermalization is confirmed by inspecting the time evolution of the occupation in the upper band $f_{+}(\mathbf{k} ; t)=\boldsymbol{\phi}_{\mathbf{k},+}^{\dagger} \boldsymbol{\rho}(\mathbf{k} ; t) \boldsymbol{\phi}_{\mathbf{k},+}$ with respect to the postquench free Hamiltonian $\mathbf{h}(\mathbf{k}) \phi_{\mathbf{k}, \pm}=\varepsilon_{ \pm}(\mathbf{k}) \phi_{\mathbf{k}, \pm}$, presented in Fig. 1(a) along the path $M-\Gamma-\mathrm{X}-M$ in the BZ for $V=1.0$. Initially, the SPDM is prepared as the equilibrium state of the band insulator with dominant $E$ orbital character; quenching $M$ leads to a band inversion with preserved occupation of the $E$ orbital, which after the quench has large weight in the upper band near the center of the BZ. The subsequent relaxation due to particle-particle scattering reduces the number of excited carriers. Computing the injected energy and comparing to thermal equilibrium yields the effective temperature $T_{\text {eff }}$. The corresponding equilibrium occupation $f_{+}^{\text {eq }}\left(\mathbf{k} ; T_{\text {eff }}\right)$ is represented by the red line 


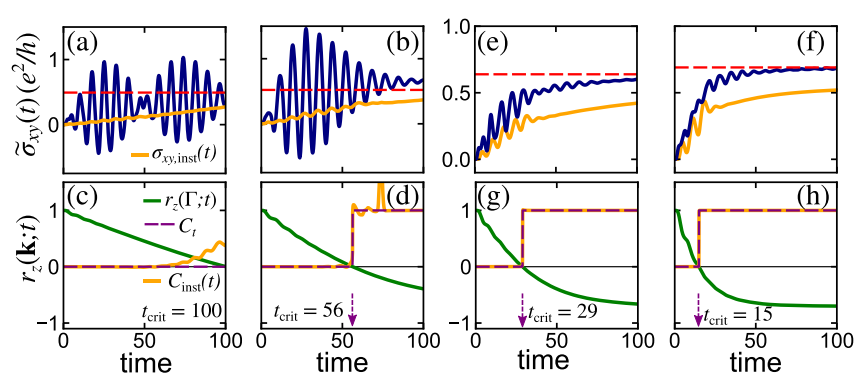

FIG. 2. Dynamics of the Hall response and orbital pseudospin for a Chern insulator with local interactions only [(a)-(d)] and with nonlocal interactions [(e)-(h)]. The interaction strength is $V=0.65$ [(a) and (c)] and $V=1.0[$ (b) and (d)] in the local case, while for the model with nonlocal interactions it is $V_{0}=0.65[(\mathrm{e})$ and $(\mathrm{g})]$ and $V_{0}=1.0$ [(f) and (h)]. We used the GKBA and show results for $N_{k}=$ $220 \times 220$ grid points in the BZ.

in Fig. 1(a). The deviation of $f_{+}(\mathbf{k} ; t)$ from $f_{+}^{\mathrm{eq}}\left(\mathbf{k} ; T_{\text {eff }}\right)$ illustrates the very slow approach to thermal equilibrium after the quench. Furthermore, the oscillations of the occupation indicate a coherent superposition of the lower and upper bands, which is only slowly damped; hence, dephasing is ineffective.

To probe the dynamical Hall response $\widetilde{\sigma}_{x y}(t)$, we apply a weak electric field $E_{y}(t)=F_{0}\left(1-e^{-t / \tau}\right)$ in the $y$ direction $\left(F_{0}=10^{-3}\right.$ and $\tau=5$ ). Measuring the induced current in the $x$ direction $J_{x}(t)$ yields the Hall response $\tilde{\sigma}_{x y}(t)=J_{x}(t) / F_{0}$. Figure 2 shows $\widetilde{\sigma}_{x y}(t)$ for $V=0.65$ (a) and $V=1.0$ (b) [also shown in Fig. 1(b)]. For $V=0.65$, strong coherent oscillations around a nonthermal value of the Hall conductance dominate and the relaxation to a steady state $\tilde{\sigma}_{x y}(t) \rightarrow \sigma_{x y}$ $(t \rightarrow \infty)$ cannot be observed on the accessible timescales. Note that a nonzero steady-state Hall conductance is a unique property of a postquench state with nontrivial topology [51]. Increasing the interaction strength to $V=1.0$, a steady state begins to form at times $t \approx 100$; however, the Hall conductance [Fig. 1(b)] does not reach the thermal equilibrium. The system remains trapped in a nonthermal state on accessible timescales, as is also evident from the nonthermal distribution [Fig. 1(a)].

Further insight into the topological properties of the SPDM can be gained by extracting the pseudospin vector $\mathbf{r}(\mathbf{k} ; \mathbf{t})$ via the relation $\rho(\mathbf{k} ; t)=[\boldsymbol{I}-\mathbf{r}(\mathbf{k} ; \mathbf{t}) \cdot \boldsymbol{\sigma}] / 2$. As long as $\mathbf{r}(\mathbf{k} ; \mathbf{t}) \neq 0$, the SPDM can formally be expressed as $\boldsymbol{\rho}(\mathbf{k} ; t)=\exp \left[-\mathbf{h}_{\text {aux }}(\mathbf{k} ; t)\right]$ with a gapped auxiliary Hamiltonian $\mathbf{h}_{\text {aux }}(\mathbf{k} ; t)$. Closing of the gap of $\mathbf{h}_{\text {aux }}(\mathbf{k} ; t)$, known from open systems as a purity gap closing [52,53], marks a dynamical topological transition at the critical time $t_{\text {crit }}$ corresponding to $\mathbf{r}\left(\Gamma ; t_{\text {crit }}\right)=0$. The occurrence of the band inversion indicated by $r_{z}(\Gamma ; t)$ passing through zero also allows one to define a simplified topological index $C_{t}$ via $(-1)^{C_{t}}=\operatorname{sgn}\left[r_{z}(\Gamma ; t) r_{z}(X ; t)\right]$. The index $C_{t}$ is identical to the Chern number $C$ of $\mathbf{h}_{\text {aux }}$ in equilibrium. This index is plotted together with $r_{z}(\Gamma ; t)$ in Figs. 2(c) and 2(d). One finds a purity gap closing at $t_{\text {crit }} \approx 100$ for $V=0.65$ and $t_{\text {crit }} \approx 56$ for $V=1.0$. Figure 1 (a) shows the distribution $f_{+}\left(\mathbf{k} ; t_{\text {crit }}\right)$, which changes its curvature at $\mathbf{k}=\Gamma$ at $t=t_{\text {crit }}$, indicating a band inversion. Further analysis [51] shows a decrease of $t_{\text {crit }}$ proportional to $V^{-3 / 2}$.
The pseudospin structure furthermore allows one to define the instantaneous Berry curvature

$$
\Omega(\mathbf{k} ; t)=-\frac{1}{2} \hat{\mathbf{r}}(\mathbf{k} ; t) \cdot\left(\frac{\partial \hat{\mathbf{r}}}{\partial k_{x}} \times \frac{\partial \hat{\mathbf{r}}}{\partial k_{y}}\right),
$$

where $\hat{\mathbf{r}}(\mathbf{k} ; t)=\mathbf{r}(\mathbf{k} ; t) /|\mathbf{r}(\mathbf{k} ; t)|$. The instantaneous Berry curvature defines the instantaneous Chern number of the $\operatorname{SPDM} C_{\text {inst }}(t)=(1 / 2 \pi) \int_{\mathrm{BZ}} d \mathbf{k} \Omega(\mathbf{k} ; t)$ and the instantaneous Hall conductance $\sigma_{x y \text {,inst }}(t)=\int_{\mathrm{BZ}} d \mathbf{k}|\mathbf{r}(\mathbf{k} ; t)| \Omega(\mathbf{k} ; t)$. Importantly, $C_{\text {inst }}(t)$ would be pinned to zero in a noninteracting system. In contrast, Figs. 2(c) and 2(d) show a nonzero instantaneous Chern number, which for $V=1.0$ becomes almost identical to $C_{t}$ [in the thermodynamic limit, $C_{t}(t)$ is identical to $\left.C_{\text {inst }}(t)\right]$. The instantaneous Hall conductance exhibits a fast increase for $t<t_{\text {crit }}$ and a saturation after $t>t_{\text {crit }}$. However, $\sigma_{x y \text {,inst }}(t)$ does not coincide with the thermalized Hall conductance of the interacting system.

Chern insulator with nonlocal interactions. The thermalization process changes substantially if nonlocal interactions are included. For the purpose of this study, we consider

$$
\hat{H}_{\mathrm{int}}=\frac{1}{2} \sum_{i, j} \sum_{\alpha, \alpha^{\prime}} V_{i, j}^{\alpha \alpha^{\prime}} \hat{n}_{i, \alpha} \hat{n}_{j, \alpha^{\prime}} .
$$

Here, the interactions are $V_{i, i}^{\alpha \alpha^{\prime}}=V_{0}\left(1-\delta_{\alpha \alpha^{\prime}}\right)$ and $V_{i, j}^{\alpha \alpha^{\prime}}=V_{1}$ if $i$ and $j$ are nearest neighbors. The nonlocal repulsion is fixed to $V_{1}=0.25 V_{0}$.

Figure 1(c) depicts the occupation of the upper band (with respect to the postquench free Hamiltonian) $f_{+}(\mathbf{k} ; t)$ for $V_{0}=$ 1.0. In contrast to the model with local interactions only, the nonlocal part of the interaction includes interorbital and intraorbital scattering, which results in a rapid thermalization of $f_{+}(\mathbf{k} ; t)$ to the equilibrium $f_{+}^{\mathrm{eq}}(\mathbf{k})$. Furthermore, coherent oscillations are suppressed, indicating pronounced dephasing. The nonequilibrium Hall response is shown in Figs. 2(e) and 2(f) for $V_{0}=0.65$ and $V_{0}=1.0$, respectively. In this setup, $\tilde{\sigma}_{x y}(t)$ approaches the thermal equilibrium value within the numerically accessible time window. Furthermore, it shows a qualitatively very similar behavior as the instantaneous conductance $\sigma_{x y \text {, inst }}(t)$, indicating strong dephasing. There are two different regimes: a rapid increase with superimposed oscillations for $t<t_{\text {crit }}$ and a smooth saturation for $t>t_{\text {crit }}$. This behavior of $\sigma_{x y \text {,inst }}(t)$ is also reflected in $\widetilde{\sigma}_{x y}(t)$. The timescale of the purity gap closing [Figs. 2(g) and 2(f)] is significantly shorter as compared to the case with local interactions only: $t_{\text {crit }} \approx 29$ for $V_{0}=0.65$ and $t_{\text {crit }} \approx 15$ for $V_{0}=1.0$. The sign of the curvature of the distribution $f_{+}(\Gamma ; t)$ changes at $t=t_{\text {crit }}$ [Fig. 1(c)]. Again, $t_{\text {crit }}$ scales as $V^{-3 / 2}$. Additional calculations [51] reveal a further speedup of $t_{\text {crit }}$ if more energy is injected by the quench.

Quantum spin Hall insulator. Including the spin degree of freedom while requiring time-reversal symmetry gives rise to a $\mathbb{Z}_{2}$ quantum spin Hall insulator. This is the typical scenario in materials where the spin-orbit interaction is the mechanism behind the topological gap opening. In this case, the on-site Hubbard repulsion (which is excluded by the Pauli principle in the spinless case) becomes the simplest possible interaction term [41]. For the sake of consistency with the previous discussion, we also include a local interorbital coupling and 


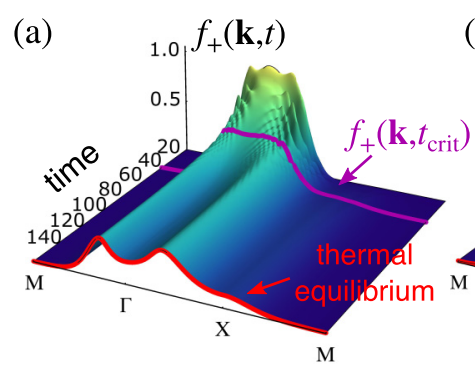

(b)
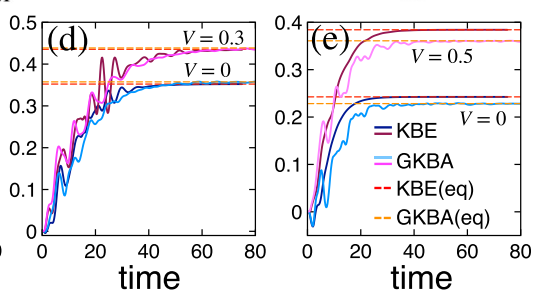

FIG. 3. Dynamics of the occupation $f_{+}(\mathbf{k} ; t)$ in the upper band of a quantum spin Hall insulator for (a) $U=1.0, V=0$, and (b) $U=$ 1.5, $V=0$. The results have been obtained employing the GKBA. The lower panels depict the nonequilibrium spin Hall conductance for (c) $U=1.0$, (d) $U=1.5$, and (e) $U=2.0$.

define the interaction term as

$$
\hat{H}_{\text {int }}=U \sum_{i, \alpha} \hat{n}_{i, \alpha, \uparrow} \hat{n}_{i, \alpha, \downarrow}+\frac{V}{2} \sum_{i, \sigma} \sum_{\alpha \neq \alpha^{\prime}} \hat{n}_{i, \alpha, \sigma} \hat{n}_{i, \alpha^{\prime}, \sigma} .
$$

We employ the same quench protocol as above and fix $M_{\text {pre }}=$ -3.5 and $M_{\text {post }}=-1.5$.

Inspecting the occupation in the upper band for weak $[U=$ 1.0, Fig. 3(a)] and slightly increased $[U=1.5$, Fig. 3(b)] Hubbard repulsion, one finds rapid dephasing and thermalization. Hence, the on-site intraorbital interaction is sufficient to fully thermalize the system on short timescales (determined by the interaction strength). Adding the interorbital coupling $V$ does not change this behavior, albeit the thermalization becomes slightly slower as $T_{\text {eff }}$ is reduced. A detailed analysis furthermore reveals a pronounced dependence on $M_{\text {post }}$ [51].

While the total Hall conductance vanishes in the spin Chern insulator, the spin Hall conductance $\widetilde{\sigma}_{x y}^{s}(t)=\left[\tilde{\sigma}_{x y}^{\uparrow}(t)-\right.$ $\left.\tilde{\sigma}_{x y}^{\downarrow}(t)\right] / 2$ becomes quantized to one at zero temperature, due to spin rotation symmetry around the $z$-axis. The spin Hall conductance is presented in Figs. 3(c)-3(e). Within the GKBA on a $N_{k}=220 \times 220$ grid, which corresponds to the converged thermodynamic limit in all cases, $\widetilde{\sigma}_{x y}^{s}(t)$ rises rapidly and approaches the thermal equilibrium value at the corresponding effective temperature $T_{\text {eff }}$. As for the spin Chern insulator, the characteristic timescale for the buildup of the spin Hall effect is the critical time $t_{\text {crit }}$ of the purity gap closing [also indicated in Figs. 3(a) and 3(b)]. Increasing the strength of the Hubbard repulsion (while keeping $V=0$ ) leads to significantly enhanced dephasing, while the steady-state spin Hall conductance $\sigma_{x y}^{s}$ is reduced. This can again be attributed to the increase of injected energy due to a stronger effective quench. Including the interorbital interaction $V$ counteracts this effect and thus results in a larger $\sigma_{x y}^{s}$. Hence, tuning the interorbital coupling $V$ provides a way of effectively cooling down the system and thus increasing $\sigma_{x y}^{s}$.
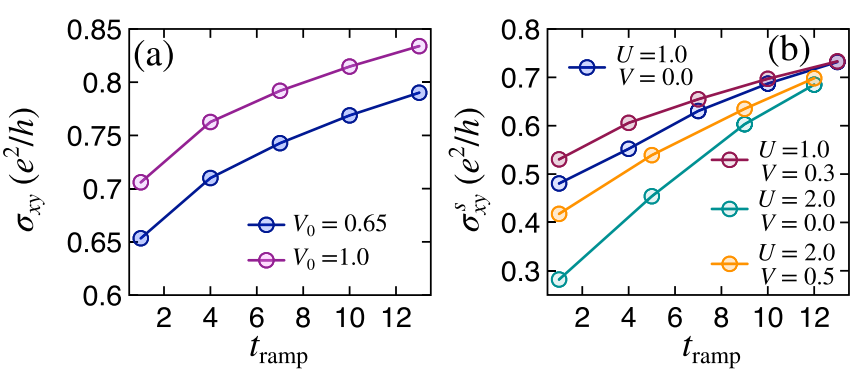

FIG. 4. Steady-state (spin) Hall conductance of (a) the spinless Chern insulator with nonlocal interactions $\left(V_{1}=0.25 V_{0}\right)$ and (b) the spinful $\mathbb{Z}_{2}$ insulator as a function of the ramp time. The GKBA has been used in (a) and (b) for all results, except for $U=2.0$, where the $\mathrm{KBE}$ result is shown.

It is interesting to compare the GKBA to the full solution of the KBEs [darker lines in Figs. 3(c)-3(e)]. Due to the numerical effort, the KBE simulations are limited to a $32 \times 32$ cluster here. For $U=1.0$, the GKBA and KBE results agree well up to $t \approx 15$; for later times, finite-size effects dominate the KBE dynamics. Nevertheless, the steady-state $\sigma_{x y}^{s}$ agrees well. For the slightly larger interaction $U=1.5$ [Fig. 3(d)], where dephasing is significantly enhanced and finite-size effects thus suppressed, the GKBA and KBE dynamics agree well, as does the steady state. For stronger interactions, however, small deviations become apparent [Fig. 3(e)]: the GKBA seems to underestimate the KBE Hall conductance. This tendency is further investigated in the Supplemental Material [51]. However, for moderate interaction strength $U \lesssim 1.5$, the GKBA and KBE treatments agree very well. Since the exact solution is typically in between [54], this comparison demonstrates the predictive power of our approach.

Realizing a low-temperature topological state. After the quench, the (spin) Chern insulator thermalizes to a state with high $T_{\text {eff }}$. The effective temperature can, however, be lowered significantly by slowly ramping $M$. We demonstrate this behavior by modifying our protocol to (i) preparing the band-insulating system in equilibrium including $\hat{H}_{\text {int }}$, and (ii) modifying the gap according to $M(t)=M_{\text {pre }}+\left(M_{\text {post }}-\right.$ $\left.M_{\text {pre }}\right) f\left(t / t_{\text {ramp }}\right)$, where $t_{\text {ramp }}$ defines the ramp duration. Here, $f(\tau)$ parametrizes the quench. For concreteness we choose the smooth $\operatorname{ramp} f(\tau)=10 \tau^{3}-15 \tau^{4}+6 \tau^{5}$ for $\tau \in[0,1]$. Figure 4(a) [Fig. 4(b)] shows the corresponding steady-state (spin) Hall conductance. As Fig. 4 demonstrates, the finite-temperature Hall effect can be significantly enhanced for all interactions by increasing $t_{\text {ramp }}$. Slower ramps lead to an adiabatic time evolution for more and more points in the BZ except for the region close to the gap closing. Therefore, the ability of the system to thermalize results in an arbitrarily low $T_{\text {eff }}$ [51] with $\sigma_{x y}$ or $\sigma_{x y}^{s}$ approaching the quantized value. Note that, however, the Hall response never exactly reaches the quantized value, as the time evolution is nonadiabatic at the $\Gamma$ point regardless of the quench duration.

Conclusions. We have systematically investigated the postquench dynamics of closed interacting two-dimensional topological insulators. We showed that the system can dynamically approach thermal equilibrium with respect to the topological properties of the SPDM and the Hall response, even though the topological invariant of the many-body state 
stays pinned to the trivial value. Thus, our results demonstrate that the eigenstate thermalization hypothesis also applies to topologically constrained unitary time evolution. The microscopic scattering mechanisms play a crucial role: while the spinless Chern insulator with local interactions only is nonintegrable, it thermalizes very slowly due to topological restrictions. In contrast, including intraorbital coupling by nonlocal interactions or extending to a spinful $\mathbb{Z}_{2}$ topological insulator accelerates the thermalization on the one- and twoparticle levels. Therefore, switching from the topologically trivial to the nontrival regime by slow ramps allows one to realize a low-temperature state with almost integer Hall conductance, providing a way of dynamically inducing and observing topological phenomena.

Acknowledgments. This work was supported by the Swiss National Science Foundation through NCCR MARVEL and the European Research Council through ERC Consolidator Grant No. 724103. The calculations have been performed on the Beo05 cluster at the University of Fribourg, and the Piz Daint cluster at the Swiss National Supercomputing Centre (CSCS). M.S. acknowledges financial support by the Alexander von Humboldt Foundation. J.C.B. acknowledges financial support from the German Research Foundation (DFG) through SFB 1143 (Project-Id 247310070).
[1] M. Aidelsburger, M. Atala, M. Lohse, J. T. Barreiro, B. Paredes, and I. Bloch, Phys. Rev. Lett. 111, 185301 (2013).

[2] H. Miyake, G. A. Siviloglou, C. J. Kennedy, W. C. Burton, and W. Ketterle, Phys. Rev. Lett. 111, 185302 (2013).

[3] G. Jotzu, M. Messer, R. Desbuquois, M. Lebrat, T. Uehlinger, D. Greif, and T. Esslinger, Nature (London) 515, 237 (2014).

[4] Z. Wu, L. Zhang, W. Sun, X.-T. Xu, B.-Z. Wang, S.-C. Ji, Y. Deng, S. Chen, X.-J. Liu, and J.-W. Pan, Science 354, 83 (2016).

[5] N. Fläschner, B. S. Rem, M. Tarnowski, D. Vogel, D.-S. Lühmann, K. Sengstock, and C. Weitenberg, Science 352, 1091 (2016).

[6] N. Goldman, J. C. Budich, and P. Zoller, Nat. Phys. 12, 639 (2016).

[7] Y. H. Wang, H. Steinberg, P. Jarillo-Herrero, and N. Gedik, Science 342, 453 (2013).

[8] J. W. McIver, B. Schulte, F.-U. Stein, T. Matsuyama, G. Jotzu, G. Meier, and A. Cavalleri, arXiv:1811.03522.

[9] M. Claassen, D. M. Kennes, M. Zingl, M. A. Sentef, and A. Rubio, Nat. Phys. 15, 1 (2019).

[10] Q. Niu, D. J. Thouless, and Y.-S. Wu, Phys. Rev. B 31, 3372 (1985).

[11] K. Kudo, H. Watanabe, T. Kariyado, and Y. Hatsugai, Phys. Rev. Lett. 122, 146601 (2019).

[12] This is different from symmetry-protected topological invariants, where dynamical symmetry breaking can occur due to a quench [55].

[13] X. Chen, Z.-C. Gu, and X.-G. Wen, Phys. Rev. B 82, 155138 (2010).

[14] D. T. Tran, A. Dauphin, A. G. Grushin, P. Zoller, and N. Goldman, Sci. Adv. 3, e1701207 (2017).

[15] M. Schüler and P. Werner, Phys. Rev. B 96, 155122 (2017).

[16] D. T. Tran, N. R. Cooper, and N. Goldman, Phys. Rev. A 97, 061602(R) (2018).

[17] C. Repellin and N. Goldman, Phys. Rev. Lett. 122, 166801 (2019).

[18] L. Asteria, D. T. Tran, T. Ozawa, M. Tarnowski, B. S. Rem, N. Fläschner, K. Sengstock, N. Goldman, and C. Weitenberg, Nat. Phys. 15, 449 (2019).

[19] M. D. Caio, N. R. Cooper, and M. J. Bhaseen, Phys. Rev. Lett. 115, 236403 (2015).

[20] H. Dehghani and A. Mitra, Phys. Rev. B 92, 165111 (2015).
[21] H. Dehghani, T. Oka, and A. Mitra, Phys. Rev. B 91, 155422 (2015).

[22] P. Wang, M. Schmitt, and S. Kehrein, Phys. Rev. B 93, 085134 (2016).

[23] Y. Hu, P. Zoller, and J. C. Budich, Phys. Rev. Lett. 117, 126803 (2016).

[24] M. Schmitt and P. Wang, Phys. Rev. B 96, 054306 (2017).

[25] L. Ulčakar, J. Mravlje, A. Ramšak, and T. Rejec, Phys. Rev. B 97, 195127 (2018).

[26] L. Peralta Gavensky, G. Usaj, and C. A. Balseiro, Phys. Rev. B 98, 165414 (2018).

[27] Y. Xu and Y. Hu, Phys. Rev. B 99, 174309 (2019).

[28] S. Wolff, A. Sheikhan, and C. Kollath, Phys. Rev. A 94, 043609 (2016).

[29] J. Berges, S. Borsányi, and C. Wetterich, Phys. Rev. Lett. 93, 142002 (2004).

[30] M. Moeckel and S. Kehrein, Phys. Rev. Lett. 100, 175702 (2008).

[31] M. Eckstein, M. Kollar, and P. Werner, Phys. Rev. Lett. 103, 056403 (2009).

[32] M. Marcuzzi, J. Marino, A. Gambassi, and A. Silva, Phys. Rev. Lett. 111, 197203 (2013).

[33] L. D’Alessio, Y. Kafri, A. Polkovnikov, and M. Rigol, Adv. Phys. 65, 239 (2016).

[34] A. Kruckenhauser and J. C. Budich, Phys. Rev. B 98, 195124 (2018).

[35] K. Balzer and M. Bonitz, Nonequilibrium Green's Functions Approach to Inhomogeneous Systems (Springer, New York, 2012).

[36] G. Stefanucci and R. v. Leeuwen, Nonequilibrium Many-Body Theory of Quantum Systems: A Modern Introduction (Cambridge University Press, Cambridge, UK, 2013).

[37] B. A. Bernevig, T. L. Hughes, and S.-C. Zhang, Science 314, 1757 (2006).

[38] C.-L. Hung, V. Gurarie, and C. Chin, Science 341, 1213 (2013).

[39] P. Makotyn, C. E. Klauss, D. L. Goldberger, E. A. Cornell, and D. S. Jin, Nat. Phys. 10, 116 (2014).

[40] M. Hohenadler, T. C. Lang, and F. F. Assaad, Phys. Rev. Lett. 106, 100403 (2011).

[41] J. C. Budich, B. Trauzettel, and G. Sangiovanni, Phys. Rev. B 87, 235104 (2013).

[42] A. Amaricci, J. C. Budich, M. Capone, B. Trauzettel, and G. Sangiovanni, Phys. Rev. Lett. 114, 185701 (2015). 
[43] A. Amaricci, A. Valli, G. Sangiovanni, B. Trauzettel, and M. Capone, Phys. Rev. B 98, 045133 (2018).

[44] H. Aoki, N. Tsuji, M. Eckstein, M. Kollar, T. Oka, and P. Werner, Rev. Mod. Phys. 86, 779 (2014).

[45] K. Balzer, N. Schlünzen, and M. Bonitz, Phys. Rev. B 94, 245118 (2016).

[46] M. Schüler and Y. Pavlyukh, Phys. Rev. B 97, 115164 (2018).

[47] P. Lipavsky, V. Spicka, and B. Velicky, Phys. Rev. B 34, 6933 (1986).

[48] S. Latini, E. Perfetto, A.-M. Uimonen, R. van Leeuwen, and G. Stefanucci, Phys. Rev. B 89, 075306 (2014).

[49] E. Perfetto, A.-M. Uimonen, R. van Leeuwen, and G. Stefanucci, Phys. Rev. A 92, 033419 (2015).
[50] E. V. n. Boström, A. Mikkelsen, C. Verdozzi, E. Perfetto, and G. Stefanucci, Nano Lett. 18, 785 (2018).

[51] See Supplemental Material at http://link.aps.org/supplemental/ 10.1103/PhysRevB.100.041101 for numerical details and additional analysis.

[52] S. Diehl, E. Rico, M. A. Baranov, and P. Zoller, Nat. Phys. 7, 971 (2011).

[53] C.-E. Bardyn, L. Wawer, A. Altland, M. Fleischhauer, and S. Diehl, Phys. Rev. X 8, 011035 (2018).

[54] N. Schlünzen, J.-P. Joost, F. Heidrich-Meisner, and M. Bonitz, Phys. Rev. B 95, 165139 (2017).

[55] M. McGinley and N. R. Cooper, Phys. Rev. Lett. 121, 090401 (2018). 